\title{
Regard diachronique et prospectif sur un demi- siècle de traductologie pragmatique
}

Translation studies in retrospect: from pioneer theories to translation ergonomics and machine translation

\section{Élisabeth Lavault-Olléon}

\section{CpenEdition}

\section{Journals}

Édition électronique

URL : http://journals.openedition.org/asp/5239

DOI : 10.4000/asp.5239

ISSN : 2108-6354

Éditeur

Groupe d'étude et de recherche en anglais de spécialité

\section{Édition imprimée}

Date de publication : 1 novembre 2018

Pagination : 5-26

ISSN : $1246-8185$

Référence électronique

Élisabeth Lavault-Olléon, « Regard diachronique et prospectif sur un demi-siècle de traductologie pragmatique », ASp [En ligne], 74 | 2018, mis en ligne le 01 novembre 2019, consulté le 01 novembre 2020. URL : http://journals.openedition.org/asp/5239; DOI : https://doi.org/10.4000/asp.5239

Ce document a été généré automatiquement le 1 novembre 2020

Tous droits réservés 


\title{
Regard diachronique et prospectif sur un demi-siècle de traductologie pragmatique
}

Translation studies in retrospect: from pioneer theories to translation ergonomics and machine translation

\author{
Élisabeth Lavault-Olléon
}

1 La diachronie en traduction peut renvoyer aux modifications des pratiques à travers les âges, au développement des théories fondées sur ces pratiques, à la comparaison des traductions d'une même œuvre au fil des siècles ainsi qu'à l'évolution des écrits euxmêmes, de leur acceptabilité et de l'interprétation de leur contenu, y compris dans les domaines de spécialité. Elle prend en compte l'évolution des langues, que ce soit en terminologie, en phraséologie ou en morphosyntaxe, mais aussi celle des idées et des normes sociales et artistiques. La discipline récente qu'est la traductologie se penche sur toutes ces questions, notamment sur le traitement de la temporalité en traduction, dont l'analyse peut souligner, par exemple, une démarche sourcière plus littéraliste tendant à marquer la distance temporelle entre l'œuvre et son lecteur, ou bien une démarche cibliste prônant l'adaptation au lecteur d'aujourd'hui (Ladmiral 2015).

2 En cette année 2018 où l'on célèbre le cinquantenaire d'événements qui ont marqué l'université française, on peut aussi évoquer la création du néologisme « traductologie » en 1968 ainsi que la première publication française ayant pour objet le processus de traduction, plus précisément d'interprétation, abordé dans une perspective communicative et pragmatique (Seleskovitch 1968). Nous en ferons donc le point de départ d'un balayage diachronique coloré par notre travail d'enseignantechercheuse et par l'orientation pragmatique qui va de pair avec notre engagement dans la formation de traductrices et traducteurs.

Notre pragmatisme est d'abord une approche concrète des phénomènes traductifs, qui considère la traduction comme une action née d'une situation définie et produisant un résultat et des effets. Il s'inscrit aussi dans le courant pragmatique de la linguistique en privilégiant l'usage et les usagers de la langue, et donc le contexte de l'opération 
traduisante (et des documents traduits) ainsi que l'ensemble des acteurs impliqués dans le processus, du commanditaire au lecteur. Des traductologues (Baker 1992 ; Froeliger 2013, entre autres) mettent en avant cette approche pragmatique dans l'étude de la traduction. Comme le constate Nicolas Froeliger, qui loue d'ailleurs l'emploi polysémique du mot "pragmatique», "une traductologie se voulant pragmatique présente donc l'intérêt de s'ouvrir à la dimension extralinguistique et communicative de l'opération de traduction » (Froeliger 2013 : 221). Cette approche s'appuie en priorité sur la traduction des textes dits pragmatiques, "ceux qui servent essentiellement à véhiculer une information et dont l'aspect esthétique n'est pas l'aspect dominant " (Delisle 1980:22), mais elle pourrait aussi s'appliquer à des genres littéraires (le théâtre, notamment). Dans notre cas, la traductologie pragmatique prend pour objet les pratiques de traduction spécialisée et a des liens évidents avec la recherche en langues et cultures de spécialité. "Traduction spécialisée » est le terme employé dans les formations de traducteurs en France pour désigner la traduction de textes pragmatiques en insistant sur leur ancrage dans des domaines de spécialité (droit, technologie, médecine, par exemple), excluant notamment les textes de la presse générale.

4 Nous rappellerons, dans un premier temps, la genèse et le développement de la traductologie jusqu'à son autonomie, puis les différentes orientations des théories fondatrices, et nous aborderons, en troisième partie, les dernières évolutions qui nous tiennent particulièrement à cœur: la démarche ergonomique et les enjeux liés aux progrès de l'automatisation.

\section{La naissance de la traductologie}

\section{De la traduction à la traductologie}

5 À l'origine était la traduction, née de la diversification des langues et du contact des cultures, " partie intégrante de la vie intellectuelle de tout peuple civilisé » (Newmark 1982: 366). Elle regroupe des pratiques multiples qui ont laissé leurs traces dans l'Histoire, sous forme d'œuvres littéraires et de textes sacrés traduits et retraduits, mais aussi de documents variés, commerciaux, administratifs, juridiques, scientifiques, par exemple, dont la traduction a permis la circulation des idées et des marchandises dans le monde et la consolidation des États multilingues : les traductions des œuvres d'Homère ou celles de la Bible ont ainsi jalonné l'histoire de la traduction et animé ses controverses, mais l'inscription qui figure sur la pierre de Rosette, le plus célèbre texte trilingue de l'antiquité égyptienne, est un décret établi à la gloire de Ptolémée V pour renforcer son pouvoir politique. La composante «spécialisée » de la traduction a bien toujours été présente, dans l'ombre de traductions plus universelles et plus souvent mises en valeur.

De Cicéron à Walter Benjamin ${ }^{1}$, en passant par Saint-Jérôme et Étienne Dolet, l'activité de traduction a généré une importante production d'écrits émanant de sources diverses : des lecteurs, des critiques ou éditeurs et, en premier lieu, des traducteurs ${ }^{2}$, qui ont écrit des préfaces ou des lettres pour justifier et expliquer leurs choix. Il existe ainsi un abondant corpus d'écrits sur la traduction s'inscrivant dans des disciplines telles que la littérature comparée, la critique littéraire, la théologie et l'herméneutique, ainsi que la stylistique et la linguistique contrastive à la recherche de points de contact 
entre les langues. Mais tout cela ne constitue pas une discipline, d'autant que ces écrits gardent leur ancrage disciplinaire. Ceux des traducteurs sont parfois difficiles à classer, mais leur caractère subjectif, hétérogène et morcelé ne suffit pas non plus à construire une discipline traductologique, qui serait tout simplement la discipline qui prend la traduction pour objet d'étude.

7 C'est à l'université que se créent et se développent les disciplines qui peuvent être, comme en France, reconnues par les instances universitaires nationales. Dans celles-ci, la traductologie n'a pas tout à fait gagné ses lettres de noblesse en France puisqu'elle n'est pas présente dans les 77 disciplines répertoriées par le Conseil national des universités. Notre expérience nous permet d'avancer qu'une raison de cette absence pourrait être le clivage des traductologues entre les deux pôles que sont les Sciences du langage d'une part et les Langues et littératures d'autre part. Mais l'abondance d'articles et d'ouvrages en traductologie, les sociétés savantes la prenant pour objet, l'introduction de cours intitulés Traductologie, les postes d'enseignants-chercheurs ciblés sur cette discipline en attestent clairement l'existence et la richesse ${ }^{3}$.

\section{Une discipline liée aux besoins de formation}

8 En prenant pour indicateur la création des grandes écoles de traduction francophones, on peut avancer que la genèse de cette discipline est le fruit d'une conjoncture sociopolitique et universitaire internationale datant de la Seconde Guerre mondiale. L'essor de la diplomatie et des négociations entre les États, comme en témoigne notamment la création de l'ONU (1945) et des grandes organisations internationales actuelles, a nécessité de mettre en place une véritable formation d'interprètes et de traducteurs performants dans ces contextes. Cela a conduit, par exemple, à la création de l'École de traduction et d'interprétation de Genève (ETI) dès 1941. L'après-guerre a également coïncidé avec le redémarrage des échanges internationaux qui ont progressivement conduit à la mondialisation que nous connaissons aujourd'hui. Les besoins en traduction et en interprétation ont explosé, menant à la formation d'écoles qui se sont installées le plus souvent dans les universités: par exemple, l'École supérieure d'interprètes et de traducteurs (ESIT), créée en 1957, a été rattachée à l'université de Paris. Un an plus tard, en 1958, l'Institut supérieur de traducteurs et interprètes (ISTI) est fondé à Bruxelles. Des formations universitaires à la traduction spécialisée de niveau master ont suivi à partir des années $1980^{4}$. Dans ce contexte universitaire, une recherche originale a vu le jour pour donner à ces nouvelles formations des appuis théoriques et didactiques.

Le cas de l'ESIT en France est sur ce point exemplaire : créée pour répondre au besoin de former des interprètes, l'école a recruté à la fin des années 1950 une praticienne de l'interprétation de conférence, Danica Seleskovitch. Pour mener à bien sa mission de formation, celle-ci a cherché une méthode d'enseignement en se fondant sur l'observation empirique des interprètes en action. De ses observations est née une thèse soutenue en 1973 (Seleskovitch 1975), puis une théorie qui a marqué dans la durée l'ensemble de la traductologie francophone, la théorie interprétative de la traduction (TIT), utilisée massivement dans la formation à l'ESIT et ailleurs. Ce cercle vertueux, qui va du besoin social à la formation, puis de l'observation à la théorisation pour revenir à la satisfaction du besoin, illustre bien comment cette nouvelle discipline 
a pris sa place dans les sciences humaines et sociales et s'est progressivement imposée dans les universités.

10 La création de la revue Meta, "Journal des traducteurs ", à l'université de Montréal en 1955 est un autre jalon qui marque le début de la traductologie, même si la discipline universitaire n'a été nommée en français qu'en 1968 par des chercheurs belges (Goffin 2008 : 920), puis en anglais par James Holmes (1988) dans sa conférence de 1972.

11 La discipline s'est ensuite rapidement développée, profitant d'une conjoncture très favorable à la recherche universitaire dans les années 1980. C'est ce que constatent Susan Bassnett et André Lefevere, deux traductologues issus de la littérature comparée : « the growth of Translation Studies as a separate discipline is a success story of the 1980s " (Bassnett \& Lefevere 1990: IX). L'âge de la maturité a été atteint lorsque la discipline est devenue suffisamment riche pour nourrir non seulement des encyclopédies, comme The Routledge Encyclopedia of Translation Studies (Baker 1998), et plus récemment, en ligne, le Handbook of Translation Studies (Gambier \& van Doorslaer 2010), mais aussi une ontologie (Vandepitte 2008).

\section{Un regard pragmatique sur l'article fondateur de James Holmes}

Poète américain et traducteur de poésie établi aux Pays-Bas, J. Holmes est aussi connu pour avoir écrit l'article fondateur de la traductologie issu d'une conférence donnée en 1972 et publié à titre posthume (Holmes 1988). Il y argumente le choix de la désignation «Translation Studies » et établit le périmètre de la discipline, divisée en trois branches, l'une purement théorique ("Theoretical Translation Studies»), la seconde fondée sur la description des pratiques ("Descriptive Translation Studies ») et la troisième appliquée (" Applied Translation Studies»). Le schéma reproduit ci-après a été reconstitué ultérieurement, nous en donnons une version simplifiée, adaptée et traduite par nos soins.

Figure 1. Cartographie de J. Holmes adaptée en français

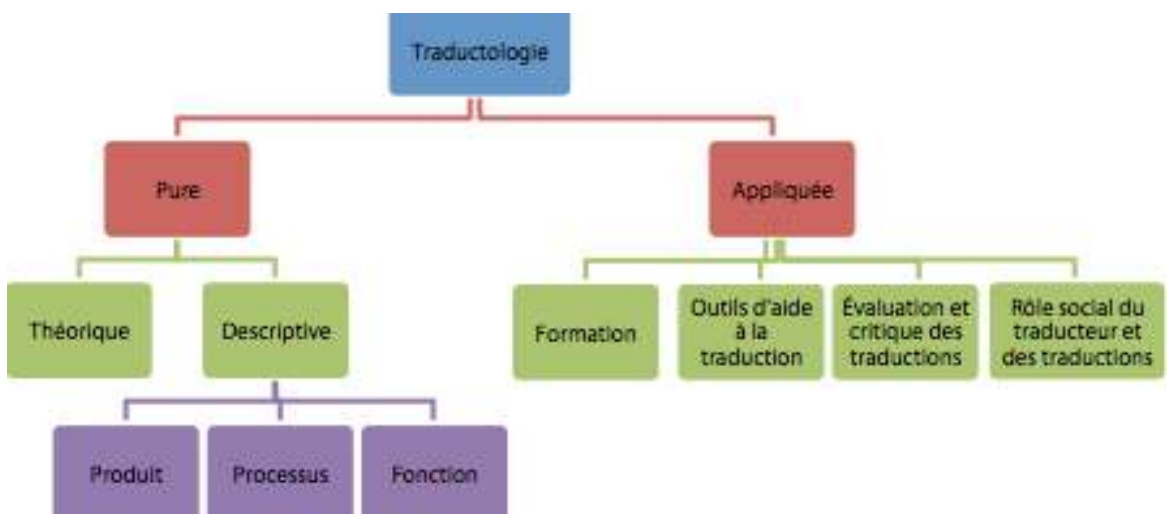

Comme annoncé plus haut, nous adoptons une perspective pragmatique en traductologie, en privilégiant les recherches sur l'action de traduire, sur ses fonctions et ses effets. La branche purement théorique ne rentre pas dans notre champ. Elle est d'ailleurs finalement assez peu représentée car la plupart des théoriciens s'appuient sur une description des pratiques. C'est bien la traductologie descriptive qui a été la plus productive, formalisée par Gideon Toury (1995). Ses trois angles d'approche (produit, processus, fonction) permettent de classer assez efficacement les théories de 
la traduction que nous évoquerons plus loin. En face, la traductologie appliquée, avec ses quatre sous-branches (formation, outils, évaluation et rôle social), est encore peu développée à l'époque où $\mathrm{J}$. Holmes écrit, mais est déjà pressentie comme une traductologie d'avenir. J. Holmes a bien noté que ces deux branches (voire les trois) se nourrissent entre elles dans une relation dialectique.

Si la traductologie appliquée est ouvertement pragmatique puisqu'elle vise une application et un résultat concrets, la traductologie descriptive nous semble également relever d'une perspective pragmatique, plus implicite. La visée pragmatique de la traductologie a été soulignée par de nombreux traductologues. Ainsi, Jean-René Ladmiral, dès 1979, défendait une réflexion ancrée dans une pratique, issue d'une pratique et élaborée pour une pratique, "une praxéologie», et notait que "le seul bénéfice que l'on est en droit d'attendre d'une théorie de la traduction, ou traductologie, consiste à clarifier et à classer les difficultés de traduction, à les conceptualiser pour articuler une logique de la décision» (1979: 211). A. Lefevere a lui aussi explicité le lien entre théorisation et production: «the goal of the discipline is to produce a comprehensive theory which can be used as a guideline for the production of translations. » (1978: 234)

Enfin, Andrew Chesterman (2006:171) souligne ce lien entre théorie et pratique :

Le but de la traductologie est tout d'abord de construire et de développer une théorie (voire des théories) de la traduction. C'est le but, en effet, de n'importe quel champ scientifique ou académique. La motivation d'un tel champ de recherche est souvent l'espoir de pouvoir appliquer les résultats de la recherche à la solution des demandes pratiques de la vie quotidienne.

Ces demandes pratiques englobent, d'après lui, aussi bien la formation optimale des traducteurs et interprètes, l'augmentation de la qualité des traductions, la formulation de méthodes appropriées pour réaliser les tâches de traduction, que le développement d'outils, la valorisation des traducteurs dans la vie sociale, l'éthique du traducteur, etc.

Cette position renforce notre conviction qu'il existe un lien étroit entre le descriptif et l'appliqué : dans nos recherches sur les pratiques actuelles de la traduction, il nous semble en effet difficile de décrire les modifications de l'activité des traducteurs et du processus de traduction lui-même, en lien avec les ressources et outils utilisés aujourd'hui, sans être tenté d'intervenir sur la formation en se fondant sur ces recherches descriptives.

\section{La fabrique de l'autonomie}

18 La conférence initiale de $\mathrm{J}$. Holmes est prononcée au moment où la traductologie va prendre son envol comme discipline autonome. Avant les années 1970, la démarche réflexive sur la traduction restait sous l'emprise d'autres disciplines, principalement celle de la littérature, d'une part, qui tendait à voir la traduction comme un art irréductible aux normes et à la description, à rapprocher de l'herméneutique; et celle de la linguistique, d'autre part, qui poussait vers une étude scientifique et objective associée au structuralisme ${ }^{5}$. C'est à la décennie suivante que la traductologie s'affirme comme discipline autonome en s'émancipant de ses deux disciplines mères. Ainsi, Antoine Berman refuse l'intervention de « tout savoir objectivant et extérieur à celle-ci [la traduction] tel qu'en élaborent la linguistique, la littérature comparée, la poétique » 
et définit la traductologie comme « la réflexion de la traduction sur elle-même à partir de sa nature d'expérience » (Berman 1999 [1985] : 38-39).

Vingt ans plus tard, en posant la question "Qu'est-ce que la traductologie ? », Michel Ballard a voulu affirmer «l'existence de la traductologie dans la diversité de ses démarches et l'abondance de sa production». Pour lui, «le facteur commun de ces approches réside dans le besoin de parler de la traduction, pour essayer de la comprendre et même, pour certains, de mieux la transmettre comme savoir-faire " (Ballard 2006: 12).

Cette discipline autonome a évolué en confirmant certaines caractéristiques jusqu'à nos jours: une visée pragmatique indissociable du contexte humain de l'activité, une réflexion sur la culture de réception, un ancrage dans les pratiques, une ouverture à l'interdisciplinarité.

21 Son émancipation de la linguistique se construit dans la résistance aux modèles dominants, à savoir le structuralisme, la grammaire générative, le formalisme, donc dans le rejet des approches objectivistes qui ne s'intéressent ni au locuteur ni aux conditions de création des énoncés, ni même au contenu. L'école de Paris et les tenants de la théorie interprétative qui sont principalement des praticiens de la traduction, D. Seleskovitch en premier, prônent un rejet total de la linguistique. Cependant, des branches de la linguistique, novatrices dans les années 1970, apportent un appui à cette nouvelle discipline: la linguistique textuelle (avec une prise en compte large du contexte qui dépasse le mot et la phrase), la linguistique énonciative (privilégiant la situation d'énonciation, clé de voûte de la théorie interprétative), la pragmatique (soulignant les effets du discours, la part des implicites et présupposés) et la sociolinguistique apportent un cadre qui permet de replacer la traduction dans son contexte de production et de prendre en compte les acteurs humains qui la modèlent. Ces approches s'intégreront progressivement en traductologie (par exemple, Pergnier 2017 [1978]).

22 Dans les années 1980, d'autres traductologues vont privilégier une perspective culturelle de la traduction, en la définissant non pas comme un transfert entre deux langues, mais prioritairement comme une interaction entre deux cultures (SnellHornby 2006; Bassnett 1998), donnant lieu à ce qu'on a appelé le «cultural turn» en traductologie. La notion de culture est alors définie au sens anthropologique comme «l'ensemble des normes, conventions et opinions qui déterminent les comportements des membres d'une société donnée, et l'ensemble des résultats de ces comportements (architecture, institution universitaire, etc.) $»^{6}$ (Vermeer 1989: 9). Or c'est la culture cible (celle dans laquelle s'inscrit la traduction) qui est majoritairement prise en compte dans les choix et stratégies des traducteurs. La nouveauté est donc d'affirmer que, une fois traduit, un texte appartient à la culture de réception: "translations are facts of target cultures » (Toury 1995 : 29). Ce tournant culturel bouscule la notion de fidélité et renforce les rangs des ciblistes, mais la priorité accordée à la réception n'implique pas une déformation ou une trahison de l'intention de l'original, les traducteurs spécialisés qui pratiquent une traduction cibliste revendiquée le savent bien. Les théories fonctionnalistes ont explicité cette question en repensant les priorités de l'acte de traduire (voir plus loin, partie 2).

Un autre fondement de l'autonomie est la primauté accordée à la traduction comme expérience humaine et la prise en compte de la réalité des pratiques de traduction, accordant de ce fait la même importance à tous les domaines d'intervention 
professionnelle et ouvrant la réflexion aux différentes traductions spécialisées, qui représentent la majorité des travaux de traduction sur le marché mondial. La traductologie va alors s'intéresser aux genres textuels, à leurs formats et leurs styles propres, aux normes de production et d'acceptabilité des textes, aux fonctions de la traduction. Enfin, plus tardivement, les traductologues vont se rapprocher d'autres disciplines connexes, utiles à la bonne analyse des pratiques.

Cette orientation pragmatique et interdisciplinaire de la traductologie, débutée dans les années 1970 et 1980, en écho à celle de la linguistique, est analysée par Marie SnellHornby $(2006: 40)$ :

The pragmatic turn in linguistics as reflected in the speech-act theory, the rise of text-linguistics, the functional approach to language with the inclusion of its social and communicative aspects, clearly indicated the general trends of the 1970s. There was firstly the broadening of perspectives within linguistics (and other disciplines) and secondly the breakdown of barriers between the individual fields. The first led to the reorientation from the isolated concept of the linguistic sign and the abstract concept of the language system [...] to a holistic notion of the text as part of the world around, and the second trend led to an invaluable process of crossfertilization, whereby the study of language was enriched by insights from anthropology, philosophy, sociology and psychology.

\section{Les grandes orientations de la traductologie : un regard diachronique}

Notre balayage diachronique se doit de présenter les grandes orientations qui ont marqué le développement de la traductologie. La traductologie descriptive décrite par J. Holmes comprenait les approches fondées sur le produit, sur le processus et sur la fonction. Les approches axées sur le produit sont essentiellement la linguistique contrastive et la stylistique comparée, qui ont été très productives (pour le français et l'anglais, Vinay \& Darbelnet 1958 ; Chuquet \& Paillard 1987, notamment) et sont moins pertinentes en traductologie pragmatique. Après les approches fondées sur le processus et sur la fonction, nous mettrons l'accent sur une quatrième approche plus récente, fondée sur la personne qui traduit.

\section{Exemple d'une théorie fondée sur le processus : la théorie interprétative de la traduction (TIT)}

S'intéresser à ce qui se passe dans la tête du traducteur pendant l'acte de traduction était vraiment innovant dans les années 1960-1970. Et il n'est pas surprenant que ce soit une interprète de conférences, habituée à pratiquer la traduction orale et à l'observer in vivo de façon concomitante à son déroulement, qui ait publié les premières réflexions théoriques fondées sur l'observation empirique du processus de traduction. Dès ses premières recherches centrées sur le travail des interprètes en lien avec le langage et la mémoire (Seleskovitch 1968, 1975), D. Seleskovitch a mis en avant le besoin pragmatique de formation, son émancipation des modèles littéraires (en se fondant sur l'interprétation puis sur la traduction pragmatique) et le rejet de la linguistique: "L'objet de la traduction n'est pas la texture dont est faite la langue mais le sens qu'y trouve celui auquel elle s'adresse » (Seleskovitch $1976: 9$ ). Cette citation résume à elle seule la TIT, dont les principes fondamentaux se sont mis en place entre 1960 et 1975 et 
dont Colette Laplace situe la «phase de maturation " dans les années 1975-1989 (Laplace $2005: 22$ ).

Parmi ses concepts novateurs et marquants, la primauté donnée au sens implique une interprétation et une reconstruction de celui-ci en se fondant autant (voire plus) sur les éléments extralinguistiques (la situation, le contexte, mais aussi le bagage cognitif en constante augmentation du traducteur) que sur les éléments linguistiques. La notionclé d'équivalence prend un sens contextuel et dynamique similaire au sens que prônait déjà Eugene Nida en l'appliquant à la traduction biblique (Nida \& Taber 1982). Elle s'oppose aux "correspondances " linguistiques caractéristiques du littéralisme. Les ouvrages de D. Seleskovitch et Marianne Lederer $(1984 ; 1989)$ abondent d'exemples convaincants d'équivalences inédites créées dans leur pratique, le principe étant que le sens compris par le destinataire de la traduction soit équivalent au «vouloir-dire » du locuteur de l'énoncé original. Entre les deux, la notion clé de déverbalisation renforce l'idée d'un sens non verbal invariant sous l'habillage linguistique, que l'on doit "rhabiller» avec les conventions linguistiques et culturelles du destinataire. Cette notion, très contestée à l'époque, est aujourd'hui confirmée par les recherches en neurosciences ${ }^{7}$ que D. Seleskovitch avait d'ailleurs déjà sollicitées pour appuyer sa théorie. La triade " interprétation-déverbalisation-réexpression » place le traducteurinterprète en figure centrale de la communication interlangue, alors que les théories contrastivistes ne prenaient pas en compte la personne qui traduit.

La TIT a eu une influence majeure sur les traducteurs francophones, surtout dans les organisations internationales, mais elle reste mal connue dans le monde anglophone, du fait du faible nombre de publications en anglais sur cette théorie à l'époque. Elle a ouvert la voie aux recherches sur les processus cognitifs à l'œuvre en traduction. Aujourd'hui, nombreux sont les travaux du courant dit « empirique » qui s'appuient sur des enregistrements variés de traducteurs en pleine action, pour mieux comprendre ces processus et les appliquer à la formation. Nous y reviendrons un peu plus loin.

\section{Exemple d'une théorie fondée sur la fonction : la théorie du skopos}

Comme pour la TIT, ce sont des traducteurs et interprètes, également universitaires, qui ont développé les théories fonctionnalistes, en Allemagne notamment (Reiss \& Vermeer 1984 ; Vermeer 1996 ; Nord 1997), mais une bonne dizaine d'années plus tard.

Hans Vermeer a choisi d'appeler sa théorie skopostheorie à partir du mot grec skopos, traduit le plus souvent par purpose en anglais, et par «but » ou « finalité » en français. Il envisage la traduction comme une action de médiation des interactions humaines, le skopos étant le principe fondamental qui détermine l'action (le processus, les choix, les décisions) du traducteur : " translating is acting, i.e. a goal-oriented procedure carried out in such a way as the translator deems optimal under the prevailing circumstances " (Vermeer 1996 : 13).

31 L'innovation réside d'abord dans la démonstration que, dans certains cas, cette « finalité » du texte cible n'est pas forcément la même que celle qui a conduit au texte source. Le principe d'équivalence doit céder la place au principe d'adéquation au skopos du texte traduit: " It is not the source-text and/or its surface-structure which determines the target-text and/or its surface-structure, but the skopos.» (ibidem: 15) Le skopos est déterminé par la négociation avec le commanditaire de la traduction, un acteur essentiel qui était occulté dans toutes les théories précédentes. Le traducteur prend ses 
décisions stratégiques en fonction de l'objectif de la traduction, du destinataire, des connaissances et des attentes de celui-ci, modelées par sa culture.

Les concepts marquants sont la primauté de la culture de réception, le "détrônement " du texte source, qui devient une simple « offre d'informations » dans laquelle on choisit les éléments pertinents à traduire, et l'effacement de l'auteur (souvent anonyme et collectif en traduction spécialisée, de toute façon), ce qui conduit H. Vermeer à donner une définition de la traduction qui ne mentionne ni l'auteur ni le texte source: «To translate means to produce a text in a target setting for a target purpose and target addresses in target circumstances. » (Vermeer 1987, cité par Nord 1997 : 12)

Enfin, le skopos annihile la dichotomie sourcier/cibliste puisqu'un skopos peut justifier une approche cibliste et un autre une approche sourcière. La notion de qualité en est également modifiée :

[L]a réflexion théorique sur la qualité des traductions, sous l'impulsion notamment des tenants du skopos, s'est déplacée de façon marquée de la fidélité au document de départ vers l'acceptabilité du document d'arrivée. [...] En d'autres termes, on a cessé de considérer le document traduit comme une entité dépendante et on l'étudie de plus en plus en fonction de sa propre finalité. (Brunette 2007 : 229)

Cette théorie peut s'appliquer à la traduction littéraire, où l'éditeur (commanditaire) joue un rôle clé et peut influer sur le traducteur en se fondant sur les attentes de son lectorat. Des traductions différentes peuvent être simplement justifiées par leur skopos : par exemple, une traduction assez littérale d'une scène de Shakespeare pour des apprenants dans un cours de langue, une autre plus stylisée pour une édition littéraire destinée à être lue, une traduction orientée vers l'oralisation et la diction pour le théâtre et les comédiens, voire vers la psychanalyse pour un metteur en scène ayant sa propre vision de la pièce. La notion de « loyauté » à l'auteur a été ajoutée à cette théorie pour prendre en compte certaines spécificités de la traduction littéraire (Nord 1997 : 126 ; Lavault-Olléon 2006 : 22). La théorie du skopos est la plus marquante d'une série d'approches fonctionnalistes actives dans la sphère allemande, scandinave et finlandaise (Hönig \& Kussmaul 1982; Holz-Mänttäri 1984, Nord 1997). Elle s'inscrit clairement dans la traductologie pragmatique que nous explorons ici et occupe une place importante dans les cursus des masters de traduction.

\section{Le tournant interdisciplinaire et sociologique des années 1990}

Pour beaucoup de disciplines, la maturité s'accompagne d'une ouverture à d'autres disciplines, dans le but d'étendre et de renouveler le champ d'études. Dans les années 1990, outre les disciplines traditionnellement associées à la traduction comme la linguistique, la sociolinguistique ou la terminologie, la traductologie va au contact de disciplines ayant le vent en poupe, comme la sociologie, l'anthropologie culturelle, l'éthique, les études postcoloniales ou les études sur le genre. Elle élargit également son champ, dépassant l'écrit pour traiter de communication non-verbale, de traduction pour la scène ou d'interprétation en langue des signes. Dans les années 1990, on assiste à un véritable foisonnement des recherches en traductologie qui explorent un grand nombre d'aspects et de perspectives de la traduction, et il est difficile d'en faire une synthèse.

Une orientation marquante à l'approche du XXI ${ }^{e}$ siècle est l'étude des normes, règles et conventions socio-professionnelles qui sous-tendent l'activité de traduction. Sous 
l'influence de la sociologie, les traductologues creusent la question de l'acceptabilité et cherchent à évaluer le niveau de contrainte porté par les usages dominants, souvent plus implicites qu'explicites, qui déterminent les stratégies de traduction. La traduction est considérée comme un comportement déterminé socialement, "socially-contexted behaviour " (Schäffner 2010:2), comme une activité régie par des normes, "a normgoverned activity» (Toury 1995: 56). G. Toury réécrit la dichotomie sourcier-cibliste dans un contexte socio-culturel large, plaçant la traduction au centre d'un double système de normes: celles de la langue et cultures cibles auxquelles elle appartient, celles de la langue et culture sources en tant que représentation d'un texte préexistant. "Ce positionnement du curseur sur l'axe source-cible est ce que Toury décrit comme la «norme initiale », le choix entre l'adéquation et l'acceptabilité. » (Lavault-olléon \& Allignol 2014 : 13) La recherche sur les normes conduit aussi à définir les normes professionnelles et opérationnelles, établies par l'usage des praticiens (Chesterman 1997 : 65-68).

37 Avec cette orientation sociologique, la vie professionnelle devient un objet de recherche, ce qui va conduire, dans de nombreuses publications, à un recentrage sur les acteurs de la traduction, les métiers, les rôles et statuts professionnels après les années 2000.

\section{Le recentrage sur le traducteur : des Translator Studies aux Workplace Studies}

38 Les théories fonctionnalistes et les théories fondées sur le processus avaient déjà valorisé le rôle du traducteur, sans en faire pour autant un objet d'étude. En continuité avec le tournant sociologique, A. Chesterman plaide pour une sociologie de la traduction qui comprendrait trois aspects, la sociologie des traductions en tant que produit ; la sociologie du processus et la sociologie des traducteurs (Chesterman 2009: 16). Il regrette l'absence, dans la cartographie de J. Holmes d'une branche d'études qu'il appelle "Translator Studies", axée non pas sur le produit, le processus ou la fonction mais sur le traducteur lui-même. Transversale à plusieurs sous-branches des trois grandes catégories présentées par J.Holmes, cette branche de la traductologie comprendrait des aspects culturels (éthique, idéologie, tradition, histoire de la profession, traducteurs et traductrices célèbres), cognitifs (processus mentaux, normes et émotions) et sociologiques (statuts, comportements, réseaux, organisation du travail, relations aux autres et aux outils) :

Translator Studies covers research which focuses primarily and explicitly on the agents involved in translation, for instance on their activities or attitudes, their interaction with their social and technical environment, or their history and influence. (ibidem : 20)

L'histoire des traducteurs avait déjà suscité de nombreux écrits (Mounin 1955 ; Cary 1963 ; Delisle \& Woodsworth 1995, etc.). Après 2000, l'organisation de la profession dans tel pays ou tel domaine, les interactions entre les traducteurs et leur environnement social, l'importance et l'impact de la technologie deviennent des sujets de recherche. C'est alors que se développent les recherches sur l'environnement de travail (Workplace Studies) qui s'appuient sur des recherches empiriques réalisées sur le lieu de travail des traducteurs pour explorer la réalité de leurs pratiques et l'impact de l'environnement au sens large sur leur production. 
40 Fortement associées à ces recherches sur le monde professionnel, les recherches sur la professionnalisation des formations de traducteurs et traductrices ont dynamisé la didactique de la traduction en s'appuyant principalement sur la notion de compétence. L'approche par compétences s'est imposée dans les masters en traduction européens grâce au projet EMT (European Master's in Translation) lancé en 2008 par la Direction générale de la traduction à la Commission européenne (DGT). La demande en traducteurs hautement qualifiés s'étant accrue du fait des besoins grandissants des institutions de l'Union européenne et d'autres employeurs, le label EMT a été créé pour « contribuer à l'amélioration des formations en traduction dans l'Union européenne, tout en favorisant la coopération et les échanges entre les établissements d'enseignement supérieur proposant des cursus en traduction $»^{8}$. Le référentiel qui détaille les compétences nécessaires pour exercer le métier de traducteur au sein des institutions de l'UE et ailleurs a été élaboré à partir des travaux de recherche de professeurs et formateurs en traduction (Schäffner \& Adab, 2000 ; Gouadec 2002; PACTE 2005, etc.) et mis à jour en 2017. Le volet didactique est donc présent en traductologie pragmatique, comme il l'est dans les langues et cultures de spécialité.

\section{Nouveaux enjeux et perspectives actuelles}

41 Les nombreuses sources d'informations disponibles sur la profession de traducteur (les sites et forums professionnels, comme celui de la Société française des traducteurs, par exemple) nous apprennent que $80 \%$ des traducteurs sont indépendants et que ceux qui traduisent dans les domaines spécialisés du droit, de l'informatique, des techniques industrielles, des échanges commerciaux et de l'industrie des loisirs sont largement majoritaires parmi les quelque 16000 traducteurs recensés en France9. Les études sur l'environnement de travail des traducteurs et les retours d'étudiants stagiaires ou d'anciens diplômés révèlent qu'une véritable révolution des métiers de la traduction est en cours, liée aux transformations technologiques de la société. Dans l'approche pragmatique qui est la nôtre, ces modifications s'appliquent surtout aux traductions spécialisées, et c'est ce que nous allons observer, en excluant la traduction littéraire qui subit elle aussi de grands changements, à un moindre degré toutefois.

\section{L'évolution des pratiques}

Le traducteur en 2018 fait de la traduction outillée, c'est-à-dire qu'il s'appuie sur des outils de traduction assistée par ordinateur (TAO) qui améliorent sa productivité et la cohérence de ses traductions, mais qui évoluent sans cesse et rendent le poste de travail de plus en plus contraignant, requérant des compétences technologiques ou « instrumentales» avancées. Internet lui fournit des ressources foisonnantes qui nécessitent une véritable compétence en extraction de l'information. Le traducteur est soumis à l'autorité d'un commanditaire qui lui impose des conditions et des cadences difficiles, dans une situation de forte concurrence. Plus déroutant encore, pour la plupart des traducteurs spécialisés, notamment techniques, l'objet du travail lui-même, le texte à traduire, s'est transformé en une suite de segments parfois discontinus et souvent décontextualisés, passés à la moulinette de mémoires de traduction qui ellesmêmes ne sont pas exemptes d'erreurs. La "tâche du traducteur» si noblement présentée par Walter Benjamin est devenue un morcellement de tâches connexes 
(gestion de fichiers et manipulations informatiques de toutes sortes, relations constantes avec commanditaires et pairs, révision, relecture, gestion comptable et commerciale), qui s'ajoutent à l'activité cognitive exigeante inhérente à l'opération traduisante elle-même.

Dans les industries de la langue, dont font partie les métiers de la traduction, la traduction est vue comme un système complexe de production de contenus multilingues, englobant non seulement des acteurs et des compétences multiples mais aussi des interactions homme-machine et des éléments sociaux et environnementaux. Cette évolution interroge les traductologues qui ont besoin d'une grille d'analyse pour prendre en compte l'ensemble de ces éléments, y compris ce nouvel environnement professionnel. Il s'agit de conjuguer les recherches sur le processus, sur les métiers et sur l'environnement de travail.

\section{L'approche ergonomique en traductologie}

Dans les recherches sur l'environnement de travail, le «travail» recouvre l'activité traduisante essentiellement cognitive du traducteur, indissociable de ses conditions d'exercice, et de son résultat. Cette approche holistique du travail est celle de l'ergonomie, définie lors du quatrième Congrès international d'ergonomie (1969) comme « l'étude scientifique de la relation entre l'homme et ses moyens, méthodes et milieux de travail». L'Association internationale d'ergonomie (IEA) vise «la compréhension fondamentale des interactions entre les humains et les autres composantes d'un système » ainsi que l'optimisation du «bien-être des personnes » et de « la performance globale des systèmes $»^{10}$.

L'approche ergonomique en traductologie a été lancée en France en 2010 par un premier colloque à l'université de Grenoble, qui a ouvert la voie à l'exploration des nombreuses interfaces entre la traduction et l'ergonomie et a suscité des publications sur le sujet (entre autres, Brunette \& O'Brien 2011; Massey \& Ehrensberger-Dow 2011 ; Lavault-Olléon 2011, Ehrensberger-Dow \& O'Brien 2015). Un deuxième colloque en mars 2015, à Grenoble également, a contribué à nourrir cette thématique, qui a ensuite été l'objet d'ateliers dans de grands colloques internationaux en traductologie ${ }^{11}$.

L'ergonomie mise d'abord sur la prédominance du facteur humain et sur le besoin d'adapter le travail à l'homme et non le contraire. Elle met en avant la variabilité des individus et les aléas des milieux de travail, et donc l'importance des interactions, de la communication et de la coordination entre les différents acteurs, en développant une démarche holistique.

Choisir cette démarche en traductologie place le traducteur au centre des recherches, plutôt que l'outil ou les procédés de transfert linguistique et culturel. C'est cette activité humaine complexe qui est étudiée en situation, par des méthodes empiriques de type ethnographique, impliquant des enquêtes à large diffusion, des observations sur le terrain, des enregistrements de l'activité sous des formes variées : pour suivre la progression de la traduction en train de se faire, les chercheurs enregistrent les mouvements sur le clavier, les modifications sur l'écran, le suivi du regard sur ce même écran (oculométrie). La personne qui traduit peut aussi être amenée à penser à voix haute durant son activité, produisant des TAP (Think Aloud Protocols), qui apportent des indications sur les processus cognitifs à l'œuvre. 
L'approche ergonomique comprend trois volets, liés les uns aux autres : l'ergonomie physique qui s'attache au poste de travail et à son environnement (mobilier, bruit, lumière, etc.) pour observer son impact sur la santé et la qualité du travail ; l'ergonomie cognitive qui observe les processus mentaux au travail, notamment les interfaces homme-machine et l'impact des outils sur ces processus ; l'ergonomie organisationnelle qui observe l'organisation et la répartition des tâches, les nouvelles formes de travail collaboratif, leur impact sur les relations et la production, etc.

Dans le présent article, nous ne reviendrons pas en détail sur les nombreuses recherches de type ergonomique qui ont été menées récemment ${ }^{12}$. Nous soulignerons simplement quelques résultats marquants.

Les recherches en ergonomie physique ont souligné les disparités entre les traducteurs de différents statuts. Ainsi les traducteurs indépendants, qui travaillent souvent chez eux dans un lieu non dédié à leur activité, sont les plus mal équipés et les plus sujets aux problèmes de santé associés aux mauvaises postures comme les troubles oculaires et les troubles musculo-squelettiques des mains et du dos (Ehrensberger-Dow et alii 2016). Le besoin de sensibilisation à l'ergonomie et aux effets nocifs sur la santé d'un mauvais environnement de travail est fortement ressenti (Meidert et alii 2016). La Direction générale de la traduction à la Commission européenne à Bruxelles a nommé une correspondante chargée de ces questions (Peters-Geiben 2016), et des universités ont commencé à inclure ce volet dans leur formation de traducteurs, comme l'Université Grenoble Alpes.

Concernant l'ergonomie cognitive, des recherches montrent, entre autres, comment les technologies modifient ou altèrent l'activité cognitive des traducteurs. Par exemple, les traducteurs utilisent moins leur mémoire puisque les ressources en ligne agissent comme une externalisation de celle-ci. De plus, la recherche facilitée de termes ou expressions sur ces mêmes ressources en ligne interrompt le flux de pensée : la fluidité de l'expression naturelle sur l'axe syntagmatique est brisée par les intrusions permanentes de l'axe paradigmatique lors de la recherche en ligne (Pym 2011 ; Carré 2017). Des recherches récentes montrent aussi quelles sont les perceptions et représentations de ces outils par les traducteurs (Cadwell et alii 2016), ou comment la mauvaise maîtrise des outils conduit à des erreurs de traduction (Massey \& Ehrensberger-Dow 2011).

Enfin, les recherches en ergonomie organisationnelle soulignent la transformation des relations professionnelles, notamment la prédominance des agences de traduction (Kuznik \& Verd 2010) et la sous-traitance généralisée par ces mêmes agences, qui coupe les liens entre le traducteur et le commanditaire véritable de la traduction. Elles constatent que le contact direct avec les spécialistes du domaine a largement disparu : les traducteurs techniques ne travaillent quasiment plus dans les entreprises et ne sont donc plus en contact avec le monde industriel. Les tâches liées à l'automatisation, notamment la postédition (correction des sorties de traduction automatique), prennent de plus en plus d'importance. Enfin, l'essor de la traduction collaborative remet en cause les notions de compétence professionnelle et d'expertise.

3 Le constat dressé par ces études ergonomiques est mitigé en ce qui concerne l'évolution de l'activité humaine de traduction. La technologie a pourtant apporté beaucoup au poste de travail et a indéniablement renforcé la productivité, mais l'automatisation pose aujourd'hui des questions cruciales que nous développons ci-après. 


\section{Tendances et enjeux majeurs en 2018}

54 La traductologie pragmatique continue à creuser des thématiques déjà balisées et à explorer des territoires nouveaux: par exemple, les problématiques associées à la traduction depuis, vers et entre les langues non européennes; l'évolution des compétences en lien avec les pratiques, notamment en traduction spécialisée; les pédagogies innovantes inspirées par les travaux de Donald Kiraly dans le cadre de son approche socio-constructiviste de l'apprentissage, encourageant les étudiants à développer "their own self-concept as developing professionals» (Kiraly 2005 : 1103). Ces recherches sur le self-concept du traducteur s'inscrivent dans un nouveau courant à dominante psychologique en traductologie, nourri par les études sur la cognition et l'ergonomie cognitive. La métacognition, ou prise de conscience de ses propres processus cognitifs, en est un aspect, particulièrement intéressant pour la didactique de la traduction (Lavault-Olléon \& Carré 2012). Les études sur la subjectivité et les émotions en font également partie. Ainsi, un numéro de la revue Meta $(2016,61 / 1)$ interroge les «zones d'incertitude en traduction », le rôle du doute et la valeur ajoutée de l'incertitude (Froeliger 2016).

Mais l'enjeu majeur auquel sont confrontés les traducteurs d'aujourd'hui, ainsi que les traductologues et formateurs, est posé par les progrès de la traduction automatique (TA). Celle-ci avait déjà beaucoup progressé en adoptant des modèles statistiques fondés sur la fréquence de traductions préexistantes et rendus possibles par l'alignement de très grands corpus parallèles. Un nouveau pas a été franchi depuis 2015 avec la TA neuronale qui s'appuie sur l'intelligence artificielle, et donc sur les capacités d'apprentissage des moteurs de traduction, à partir des méga-données collectées par les grandes sociétés du numérique. Les progrès sont variables selon les couples de langues, laissant au traducteur humain des marges d'action dans certaines langues du moins, et ils sont également moins notables dans les domaines marqués par la subjectivité et la stylistique, comme la littérature ou le marketing, sans parler de secteurs sensibles comme la diplomatie.

Néanmoins, l'automatisation est en route et serait opérationnelle dans une dizaine d'années, comme le revendique Ray Kurzweil, qui affirmait déjà en 2011 que les «traducteurs» automatiques remplaceraient la majorité des traducteurs (désormais «biotraducteurs ») en 2029 - en exceptant la littérature, intraduisible de toute façon, selon lui : "even the best translators can't fully translate literature $»^{13}$. L'un des arguments avancés par les défenseurs de la TA est que la machine se trompe moins que les humains, selon les modèles normatifs qu'ils élaborent, ce qui pose la question de la légitimité du discours sur la traduction, un enjeu traductologique fondamental. L'argument a longtemps fait sourire tous ceux qui se gaussaient des absurdités des productions automatisées mais qui doivent aujourd'hui reconnaitre les progrès spectaculaires en cours puisque, justement, c'est bien sur la base de traductions humaines que la machine neuronale « apprend » à traduire.

Même si les besoins en traduction multilingue sont si énormes et croissants qu'ils peuvent être traités en grande partie par la TA sans encore menacer l'emploi des traducteurs, nous ne sommes plus très loin d'une traduction de qualité acceptable qui serait accessible à tous instantanément. Chacun a déjà pu faire l'expérience de problèmes de communication à l'étranger, à la pharmacie ou au poste de police, réglés dorénavant en quelques clics grâce au «traducteur » du téléphone portable. Tout cela, 
convenons-en, est une avancée magnifique dans la communication entre les personnes et les peuples du monde entier. C'est aussi l'amorce de la disparition d'un métier.

Comment la traductologie peut-elle rendre compte de ces bouleversements? Les traductologues ne sont pas des experts du fonctionnement des systèmes de TA, mais ils peuvent observer et décrire les usages actuels en se concentrant sur les aspects humains, dans le cadre de l'approche ergonomique déjà mentionnée. Parmi ceux-ci, par exemple, le développement de la postédition, étudiée du point de vue des perceptions qu'en ont les traducteurs et de son impact sur leur activité (Moorkens \& O'Brien 2013) ou les représentations des traducteurs sur la TA comme outil intégré au poste de travail, parfois imposé par les institutions (Rossi \& Chevrot, à paraître 2019).

L'accès généralisé à une traduction automatisée et le développement de la traduction et de la postédition collaboratives, effectuées par des personnes qui ne sont pas formées à la traduction, entraînent un nouveau partage des savoir-faire (Pym 2011) et remettent en cause l'expertise professionnelle du traducteur, au profit des experts (non linguistes) des domaines spécialisés, mais aussi des informaticiens et linguistes spécialisés dans le traitement des langues naturelles (TAL) qui possèdent le pouvoir technologique. Cette modification de sa position centrale et auparavant indispensable modifie son statut. Trouver sa valeur ajoutée devient une nécessité professionnelle, en se consacrant à des tâches spécialisées de pré-édition (préparation des textes avant l'automatisation) ou de postédition, en apportant une expertise linguistique en position de validation et d'assurance qualité, mais aussi une compétence culturelle et interculturelle encore difficile à inculquer à la machine.

Un autre objet d'étude, plus sociolinguistique, est la réflexion sur la qualité acceptable d'une traduction, et, par ricochet, sur la qualité acceptable des écrits. Nous sommes tous conscients que notre seuil d'acceptabilité en ce qui concerne la qualité de la langue a baissé face à la banalisation des écrits fautifs en termes d'orthographe et de syntaxe. Cette variation de la norme linguistique, qui rend de plus en plus acceptables des textes imparfaits traduits rapidement ou automatiquement, conduit à s'interroger sur une sorte de banalisation de la communication automatisée, et sur la simplification de la langue et de la pensée qu'elle induit. Il est grand temps de relire George Orwell, en découvrant par la même occasion la dernière traduction française de 1984.

61 La description de ces modifications a de quoi alimenter la traductologie descriptive et ses principes théoriques pendant quelque temps. La transformation du poste de travail rend en effet caduques les théories de la traduction fondées sur le schéma : émetteur $\rightarrow$ traducteur $\rightarrow$ destinataire. Comment appliquer la théorie interprétative s'il n'y a plus de contexte ni de situation fournis avec le texte à traduire, parfois réduit à l'état de fragments? Comment "interpréter le sens" s'il n'y a aucun élément extralinguistique? De même, comment appliquer la théorie du skopos s'il n'y a pas de négociation possible avec le commanditaire réel de la traduction, du fait de l'enchaînement des intermédiaires dû à la sous-traitance ? Les traductologues qui ont été nourris à ces théories assistent avec désarroi à une réduction de l'activité traduisante au transfert linguistique le plus littéral, sans aide contextuelle pour résoudre l'ambiguïté ou les erreurs présentes dans le texte à traduire.

Cette traversée de près d'un demi-siècle de traductologie si riche et intense nous amène à une conclusion plutôt sombre : les traductologues devront apprendre à survivre à la disparition programmée de la majorité des «biotraducteurs », mais ils ont encore des années d'activité pour analyser les bouleversements actuels et préparer leur mutation. 
En attendant, c'est l'importance des spécificités humaines, de la médiation interculturelle, de la sensibilité linguistique, de la subtilité des émotions, mais aussi les compétences en gestion de projets multilingues, qui donneront une valeur ajoutée aux traducteurs et de bons sujets d'étude aux traductologues. C'est aussi l'appropriation de la traduction automatique qui assurera la survie des uns et des autres dans un premier temps. À tous de savoir s'adapter, évoluer, muter vers ce que seront les nouveaux enjeux de la communication multilingue et multiculturelle de l'avenir.

\section{BIBLIOGRAPHIE}

BAKER, Mona. 1992. In Other Words: A Coursebook in translation. Londres \& New York : Routledge.

BAKER, Mona. 1998. The Routledge Encyclopedia of Translation Studies. Londres \& New York :

Routledge.

BALLARD, Michel. 1992. De Cicéron à Benjamin : Traducteurs, traductions, réflexions. Lille : Presses universitaires de Lille.

BALLARD, Michel (dir.). 2006. Qu'est-ce que la traductologie? Arras : Artois Presses Université.

BASSNETT, Susan.1998. «The translation turn in cultural studies ». In BASSNETT, S. \& A. LEFEVERE (dir.), Constructing Cultures: Essays on Literary Translation. Clevedon : Multilingual Matters, 123-140. BASSNETT, Susan \& André LEFEVERE (dir). 1990. Translation, History and Culture. Londres : Pinter. BERMAN, Antoine. 1999 [1985]. « La traduction et la lettre ou l'auberge du lointain ». In BERMAN, A. \& alii (dir.), Les tours de Babel, essais sur la traduction. Mauvezin : Trans-Europ-Repress. Réédit. Paris : Éditions du Seuil.

BRUNETTE, Louise. 2007. « Relecture-révision, compétences indispensables du traducteur spécialisé ». In LAVAULT-OLLÉON, E. (dir.), Traduction spécialisée : pratiques, théories, formations. Berne : Peter Lang, 225-235.

BRUNETTE, Louise \& Sharon O'BRIEN. 2011. «Quelle ergonomie pour la pratique postéditrice des textes traduits? ». ILCEA 14 [En ligne], consulté le 18 juin 2018 <https://journals.openedition.org/ ilcea/1081>.

CADWELL, Patrick, Sheila CASTILHO, Sharon O'BRIEN \& Linda MITCHELL. 2016. « Human factors in machine translation and post-editing among institutional translators ». Translation Spaces 5/2, 222-243.

CARRÉ, Alice. 2017. «L'analyse des processus cognitifs comme aide à la formation initiale et au perfectionnement des traducteurs ». Thèse soutenue à l'Université Grenoble Alpes.

CARY, Edmond. 1963. Les grands traducteurs français. Genève : Georg.

CHESTERMAN, Andrew. 1997. Memes of Translation: The spread of ideas in translation theory. Amsterdam - Philadelphie : John Benjamins Publishing Company.

CHESTERMAN, Andrew. 2006. «Vers une traductologie poppérienne ». In BALLARD, M. (dir.), Qu'est-ce que la traductologie? Arras : Artois Presses Université, 171-178. 
CHESTERMAN, Andrew. 2009. " The name and nature of translator studies ». Hermes 42, 13-22. Consulté le 18 juin 2018 <http://download1.hermes.asb.dk/archive/download/Hermes-42-2chesterman_net.pdf>.

CHUQUET, Hélène \& Michel PAILLARD. 1987. Approche linguistique des problèmes de traduction. Paris : Ophrys.

DELISLE, Jean \& Judith WOODSWORTH (dir.). 1995. Les traducteurs dans l'histoire. Ottawa : Presses de l'Université d'Ottawa \& éditions UNESCO.

EHRENSBERGER-DOW, Maureen \& Sharon O'BRIEN. 2015. « Ergonomics of the translation workplace: Potential for cognitive friction ». Translation Spaces 4/1, 98-118. DOI : 0.1075/ts.4.1.05ehr.

EHRENSBERGER-DOW, Maureen, Andrea HUNZIKER HEEB, Gary MASSEY, Ursula MEIDERT, Silke NEUMANN, \& Heidrun BECKER. 2016. « An international survey of the ergonomics of professional translation ». ILCEA 27 [en ligne], consulté le 18 juin 2018 <http://journals.openedition.org/ilcea/4004>. FROELIGER, Nicolas. 2013. Les noces de l'analogique et du numérique. Paris : Les Belles Lettres. FROELIGER, Nicolas. 2016. «Quelques incertitudes sur l'incertitude ». Meta : journal des traducteurs/ Meta: Translators' Journal, 61/1, 1-1. DOI : 10.7202/1036979ar.

GAMBIER, Yves \& Luc van DOORSLAER (dir.). 2010. Handbook of Translation Studies. Amsterdam Philadelphie : John Benjamins Publishing Company.

GENTZLER, Edwin. 2001. Contemporary Translation Theories. Clevedon : Multilingual Matters.

GOFFIN, Roger. 2008. Recension de Ballard Michel (2006). Qu'est-ce que la traductologie? Meta :journal des traducteur/Meta: Translators' Journal, 53/4, 920. DOI : 10.7202/019657ar.

GOUADEC, Daniel. 2002. Profession : traducteur. Paris : La maison du dictionnaire.

HOLMES, James S. 1988. « The name and nature of translation studies ». In HOLMES, J. S., Translated! Papers on Literary Translation and Translation Studies. Amsterdam : Rodopi, 67-80.

HOLZ-MÄNTTÄRI, Justa. 1984. Translatorisches Handeln. Theorie und Methode. Helsinki : Suomalainen Tiedeakatemia.

HÖNIG, Hans G. \& Paul KUSSMAUL. 1982. Strategie der Übersetzung. Ein Lehr- und Arbeitsbuch. Tübingen : Narr.

KIRALY, Donald. 2005. « Project-based learning: A case for situated translation ». Meta : journal des traducteurs/Meta: Translators' Journal, 50/4, 1098-1111. DOI : 10.7202/012063ar.

KUZNIK, Anna \& Joan M. VERD. 2010. « Investigating real work situations in translation agencies. Work content and its components ». Hermes-Journal of Language and Communication Studies 44, 2543.

LADMIRAL, Jean-René. 1979. Traduire: théorèmes pour la traduction. Paris : Payot. rééd. 1994. Paris : Gallimard.

LADMIRAL, Jean-René. 2015. Sourciers ou cibliste : les profondeurs de la traduction. Paris : Les Belles Lettres.

LAPLACE, Colette. 2005. « La genèse de la théorie interprétative de la traduction ». In ISRAËL, F. \& M. LEDERER (dir.), La théorie interprétative de la traduction, tome I. Paris : Minard, 21-66. 
LAVAULT-OLLÉON, Élisabeth. 2006. «Le skopos comme stratégie de déblocage : dialecte et scotticité dans Sunset Song de Lewis Grassic Gibbon ». Meta : journal des traducteurs/Meta: Translators' Journal, 51/3, 504-523, consulté le 18 juin 2018 <http://id.erudit.org/iderudit/013555ar>.

LAVAULT-OLLÉON, Élisabeth. 2011. « L'ergonomie, nouveau paradigme pour la traductologie ». ILCEA 14 [en ligne], consulté le 18 juin 2018 <http://journals.openedition.org/ilcea/1078>.

LAVAULT-OLLÉON, Élisabeth \& Alice CARRÉ. 2012. «Traduction spécialisée : l'ergonomie cognitive au service de la formation ». ASp 62, 67-77. DOI : 10.4000/asp.3117.

LAVAULT-OLLÉON, Élisabeth \& Claire ALLIGNOl. 2014. « La notion d'acceptabilité en traduction professionnelle : où placer le curseur? ». ILCEA 19 [en ligne], consulté le 18 juin 2018 <http:// journals.openedition.org/ilcea/2455>.

LEFEVERE, André. 1978. «Translation studies: The goal of the discipline ». In HOLMES, J., J. LAMBERT \& R. van den BROECK (dir.), Literature and Translation: New Perspectives in Literary Studies. Louvain : Acco.

MASSEY, Gary \& Maureen EHRENBERGER-DOW. 2011. « Technical and instrumental iompetence in the translator's workplace: Using process rResearch to identify educational and ergonomic needs ». ILCEA 14 [En ligne], consulté le 18 juin 2018 <http://journals.openedition .org/ilcea/1060>.

MEIDERT Ursula, Silke NEUMANN, Maureen EHRENSBERGER-DOW \& Heidrun BECKER. 2016. « Physical ergonomics at translators' workplaces: Findings from ergonomic workplace assessments and interviews ", ILCEA 27 [en ligne], consulté le 18 juin 2018 <http://journals.openedition.org/ilcea/ 3996>.

MOORKENS, JOsS \& Sharon O'BRIEN. 2013. « User attitudes to the post-editing interface ». Proceedings of MT Summit XIV Workshop on Post-editing Technology and Practice, 19-25. Allschwil: The European Association for Machine Translation.

MOUNIN, Georges. 1955. Les Belles infidèles. Paris : Éditions des Cahiers du sud.

NEWMARK, Peter. 1982. Approaches to Translation. Oxford : Pergamon Press.

NIDA, Eugene \& Charles TABER. 1982. The Theory and Practice of Translation. Leyde, Pays-Bas : United Bible Societies.

NORD, Christiane. 1997. Translating as a Purposeful Activity - Functionalist Approaches Explained.

Manchester : St. Jerome Publishing.

ORWELL, George. 2018 [1949]. 1984. Nouvelle traduction française par Josée Kamoun. Paris :

Gallimard.

PACTE Group. 2005. « Investigating translation competence: Conceptual and methodological issues ». Meta : journal des traducteurs / Meta: Translators' Journal, 50/2, 609-619, consulté le 18 juin 2018 <http://id.erudit.org/iderudit/011004ar>.

PERGNIER, Maurice. 2017 [1978]. Les Fondements socio-linguistiques de la traduction. $3^{\mathrm{e}}$ éd. Paris : Les Belles Lettres.

PETERS-GEIBEN, Lucia. 2016. « La prévention comportementale et contextuelle : intégrer une approche ergonomique dans la formation des traducteurs ", ILCEA 27 [En ligne], consulté le 18 juin 2018 <http://journals.openedition.org/ilcea/4026>.

PYM, Anthony. 2011. « What technology does to translating ». Translation \& Interpreting 3/1, 1-9, consulté le 18 juin 2018 <http://www.trans-int.org/index.php/transint/article/ view/121>. 
REISS, Katharina \& Hans J. VERMEER. 1984. Grundlegung einer allgemeinen Translationstheorie. Tübingen : Niemeyer.

ROSSI, Caroline \& Jean-Pierre CHEVROT. À paraître 2019. « Uses and perceptions of machine translation at the European Commission ». Jostrans 31 [en ligne].

SCHÄFFNER, Christina. 2010. « Norms of translation ». In GAMBIER, Y. \& L. van DOORSLAER (dir.), Handbook of Translation Studies. Vol. 1. Amsterdam - Philadelphie : John Benjamins Publishing Company, consulté le 18 juin 2018 <https://benjamins.com /online/hts/articles/nor1>.

SCHÄFFNER, Christina \& Beverley ADAB (dir.). 2000. Developing Translation Competence. Amsterdam Philadelphie : John Benjamins Publishing Company.

SELESKOVITCH, Danica. 1968. L'interprète dans les conférences internationales, problèmes de langage et de communication. Paris : Minard.

SELESKOVITCH, Danica. 1975. Langage, langues et mémoire. Paris : Minard.

SELESKOVITCH, Danica. 1976. «Introduction ». Études de linguistique appliquée, 24, 8-12.

SELESKOVITCH, Danica \& Marianne LEDERER. 1984. Interpréter pour traduire. Paris : Didier Érudition. SELESKOVITCH, Danica \& Marianne LEDERER. 1989. Pédagogie raisonnée de l'interprétation. Paris : Didier Érudition.

SNELL-HORNBY, Mary. 2006. The Turns of Translation Studies: New Paradigms or Shifting Viewpoints? Amsterdam - Philadelphie : John Benjamins.

TOURY, Gideon. 1995. Descriptive Translation Studies and beyond. Amsterdam - Philadelphie : John Benjamins.

VANDEPITTE, Sonia. 2008. « Remapping translation studies: Towards a translation studies ontology ». Meta : journal des traducteurs/Meta: Translators' Journal, 53/3, 569-588, consulté le 18 juin 2018 <http://id.erudit.org/iderudit/019240ar>.

VERMEER, Hans J. (dir.). 1989. Kulturspezifik des translatorischen Handelns. Heidelberg : Univ. Heidelberg.

VERMEER, Hans J. 1996. A Skopos Theory of Translation. Heidelberg : Textcontext Wissenschaft. VINAY, Jean-Paul \& Jean DARBELNET. 1958. Stylistique comparée du français et de l'anglais. Paris : Didier.

\section{NOTES}

1. Nous reprenons à dessein le titre d'un ouvrage érudit de Michel Ballard qui relate l'histoire de la traduction et de la traductologie (Ballard 1992).

2. Dans le présent article, conformément aux normes du français et pour faciliter la lecture, le masculin singulier «traducteur » représente le métier, même si « 4 sur 5 traducteurs sont des femmes ", d'après le site de la Société française des traducteurs (SFT) : « Analyse des données sur les pratiques professionnelles des métiers de la traduction en 2015 ». La même règle est appliquée aux autres métiers.

3. Le nombre de revues spécialisées en est un bon indicateur. Par exemple, dans l'aire francophone et anglophone occidentale, on compte plus d'une douzaine de revues spécialisées en traductologie : Babel, Equivalences, Forum, The Interpreter and Translator Trainer, Jostrans, Meta, Des mots aux actes, Palimpsestes, Target, Traduire, Translation Spaces, Translation Studies, Translation and interpreting Studies, TTR... 
4. À l'époque, il s'agissait de DESS (diplômes d'enseignement supérieur spécialisé) qui ont été pour la plupart transformés en masters en 2004. Il y a aujourd'hui de nombreux masters en traduction dans les universités françaises, dont plus d'une vingtaine de masters de traduction professionnelle se sont regroupés dans l'AFFUMT (Association française des formations universitaires aux métiers de la traduction) et sont décrits sur son site: <https:// affumt.wordpress.com>.

5. Sur ce dernier point, Edwin Gentzler (2001) propose une analyse montrant le lien (sur les structures profondes proches d'une certaine universalité du sens), puis la rupture (sur la pragmatique) entre Noam Chomsky (le linguiste) et Eugene Nida (le traductologue).

6. « die Gesamtheit der Normen, Konventionen und Meinungen, nach denen sich das Verhalten der Mitglieder einer Gesellschaft richtet, und die Gesamtheit der Resultate aus diesem Verhalten (also z.B. der architektonischen Bauten, der universitaren Einrichtungen, usw.).» (Vermeer 1989 : 9)

7. Dans les cours auxquels nous avons assisté, D. Seleskovitch mentionnait les zones du cerveau responsables du traitement différencié du langage, "l'aire de Broca » et "l'aire de Wernicke », pour appuyer sa distinction entre le sens conceptuel et ses possibles formulations linguistiques. Depuis, le cerveau a été entièrement cartographié, montrant des centaines de subdivisions fonctionnelles spécialisées et interconnectées. Voir par exemple le cours de Stanislas Dehaene, professeur de psychologie cognitive expérimentale au Collège de France "Représentation cérébrale des structures linguistiques » consulté le 7/8/18 < https://www.college-de-france.fr/ media/stanislas-dehaene/

UPL6434872702326906363_Cours2015_Representation_cerebrale_des_structures_linguistiques_2.pdf>.

8. Descriptif du Master européen en traduction (EMT) sur le site de la Direction générale de la traduction, disponible sur <https://ec.europa.eu/info/resources-partners/european-masterstranslation-emt_en>.

9. Chiffres émis par la Société française des traducteurs (SFT) : «Analyse des données sur les pratiques professionnelles des métiers de la traduction en $2015 »$, disponibles sur < https:// www.sft.fr>.

10. Ces définitions se trouvent sur le site de la Société d'ergonomie de langue française (SELF) : $<$ http://ergonomie-self.org>.

11. Par exemple, au congrès de l'EST, European Society for Translation Studies, à Aarhus en 2016, et au Congrès mondial de traductologie à Paris en 2017.

12. Voir à ce sujet les deux numéros de la revue ILCEA (en ligne) qui leur sont consacrés :

le numéro 14 (2011) <https://journals.openedition.org/ilcea/1031> et le numéro 27 (2016) $<$ https://journals.openedition.org/ilcea/3834>.

13. Voir l'interview de Ray Kurzweil, auteur de nombreux ouvrages sur la robotisation et l'automatisation, dans le Huffington Post (2011) : <https://www.huffingtonpost.com/nataly-kelly/ ray-kurzweil-on-translati_b_875745.html?guccounter=1>.

\section{RÉSUMÉS}

Depuis la création du néologisme «traductologie » en 1968, la traductologie a progressivement acquis son autonomie comme discipline universitaire et a considérablement prospéré, foisonnant en une multitude d'approches théoriques et appliquées tout en se nourrissant des apports d'autres sciences humaines. Nous nous inscrivons dans la thématique diachronique du présent 
numéro d'ASp en retraçant les grandes étapes de ce parcours de cinquante ans et plus dans une perspective pragmatique. Nous abordons ensuite les approches des dernières années et leur évolution incertaine en lien avec la traduction automatique.

Since the French term "traductologie" was coined in 1968 (and "Translation Studies" in 1972), Translation Studies has gradually acquired its autonomy as an academic discipline and has expanded in a wide variety of approaches, either theoretical or applied, in a process of crossfertilization with other human sciences. The diachronic theme of this ASp issue provides us with an opportunity to describe the major stages of this journey of over fifty years from a pragmatic perspective and then to introduce the latest research in this field and its uncertain future in relation with machine translation.

\section{INDEX}

Mots-clés : diachronie, ergonomie, traduction spécialisée, traductologie

Keywords : diachrony, ergonomics, specialized translation, translation studies

\section{AUTEUR}

\section{ÉLISABETH LAVAULT-OLLÉON}

Élisabeth Lavault-Olléon est professeure émérite à l'Université Grenoble Alpes, où elle a été responsable d'un groupe de recherche, le GREMUTS (Groupe de recherche multilingue en traduction spécialisée) intégré dans le laboratoire ILCEA4. Elle a créé à Grenoble une formation de traducteurs, le Master « Traduction spécialisée multilingue », qu'elle a dirigé jusqu'à 2017. Elle est l'auteure de nombreuses publications sur la didactique de la traduction, la traduction spécialisée, la professionnalisation, les liens entre théorie et pratique, notamment Fonctions de la traduction en didactique des langues (Didier Érudition 1985, 2e éd. 1998), Traduction spécialisée : pratiques, théories, formations (Peter Lang 2007) et a dirigé les numéros 14 et 27 de la revue ILCEA (en ligne) consacrés à la démarche ergonomique en traduction. Elisabeth.Lavault@univ-grenoblealpes.fr 